\title{
Kikuchi-Fujimoto Disease: A Rare Cause of Lymphadenopathy
}

\author{
S. Anuradha ${ }^{1}$, Vanlalmalsawmdawngliana ${ }^{1}$, Neeta Khurana ${ }^{2}$ \\ Departments of ${ }^{1}$ Medicine and ${ }^{2}$ Pathology, Maulana Azad Medical College, New Delhi -110002, India.
}

\section{Corresponding Author:}

Prof. (Dr.) S. Anuradha

Email: drsanuradha@gmail.com

This is an Open Access article distributed under the terms of the Creative Commons Attribution License (creativecommons.org/ licenses/by/3.0).

Received

Accepted

Published

December 18, 2016

March 2, 2017

March 15, 2017

\begin{abstract}
Background: Kikuchi-Fujimoto disease (KFD) also known as histiocytic necrotizing lymphadenitis is a rare and self-limiting cause of fever with lymphadenitis. It usually affects females of the reproductive age group with a predominant involvement of cervical lymph nodes. Case Report: We report a case of young boy with Kikuchi-Fujimoto disease who presented with prolonged fever and painless cervical lymphadenopathy. Conclusion: Due to numerous causes of lymphadenopathy, patients are likely to be misdiagnosed with another disease. A high clinical suspicion with histopathological confirmation is essential for early and accurate diagnosis of KFD, which is usually a self-limited condition.
\end{abstract}

Keywords: Histiocytic Necrotizing Lymphadenitis, Lymph Nodes, Lymphadenitis, Lymphadenopathy.

\section{Introduction}

Kikuchi-Fujimoto disease (KFD) was originally described by Japanese pathologists Kikuchi and Fujimoto in 1972 [1,2]. It typically manifests as a lymphadenopathy (predominantly cervical) with constitutional symptoms like fever, lethargy and myalgia. Although it runs a benign course and resolves spontaneously within few months of the onset in most patients, some fatal cases have been reported [3]. The affected patients are usually young, less than 40 years of age, with a female preponderance. A higher prevalence of this disease is seen among the Asian population, but cases are reported across the world [4]. Clinically it can be mistaken for other diseases like lymphoma, systemic lupus erythematosus (SLE) and TB lymphadenitis [5]. The diagnosis is confirmed by histopathological examination of an affected lymph nodes (LN). Though it is a rare cause for cervical lymphadenopathy, the clinician and pathologist should be aware of this condition so that it can be diagnosed with accuracy thereby avoiding unnecessary expensive and sophisticated investigations, especially in a resource constrained country like India.

\section{Case Report}

A 14 year boy presented with low grade fever for one month duration associated with constitutional symptoms of malaise and anorexia. He denied throat pain, cough, joint pains or skin rash. On examination, he was febrile and had multiple palpable LN on both sides of the neck mainly at level II and III; maximum size was approximately $2 \times 2 \mathrm{~cm}$. The $\mathrm{LN}$ were firm, non-tender and not fixed to the underlying structures. The rest of the ear, nose and throat examination were normal and no other palpable lymph nodes or organomegaly was noted. The rest of the systemic examination was unremarkable.

A fine needle aspiration cytology (FNAC) of the affected LN was suggestive of reactive lymphadenitis. An excision biopsy of the largest cervical LN was done as fever persisted inspite of seven days of oral broad spectrum antibiotic and antipyretic treatment. The biopsy revealed histiocytic necrotizing lymphadenitis with prominent karryorhexis. Granulomas were absent and acid fast bacilli (AFB) and fungal stains 
were negative [Fig.1-3]. All others investigations like liver function tests, kidney function tests, HIV, HBsAg, anti-HCV and chest X-ray were normal. Antinuclear antibody (ANA) was positive (speckled 1+) but ds-DNA was negative. These combined clinical and histopathological findings were consistent with a diagnosis of KFD.

The fever subsided on its own after around one and half months after the disease onset. Within 6 months follow up, he was asymptomatic. He does not show any features suggestive of SLE and /or recurrence.

\section{Discussion}

KFD is a rare and benign condition which usually manifests as a febrile illness with lymphadenopathy, in which $56 \%-90 \%$ of the cases involve the cervical LN. It mainly affects the young (age $<40$ years) with a female preponderance (male: female $=1: 4$ ). Although the prevalence is higher among Asians, cases are reported across the world. The disease is usually self-limiting and has a good prognosis in most patients; spontaneous resolution usually occurs within 6 months of onset. However rare fatal cases like hemophagocytic syndrome has been occasionally reported in literature $[3,4]$.

The exact etiopathogenesis of the disease remains a mystery. It is believed to be due to excessive immune response of histiocytes to infectious agents like the Epstein-Barr virus, cytomegalovirus, herpes virus and HIV [6]. Several authors have reported an association between KFD and autoimmune diseases, particularly SLE [7]. The characteristic histopathologic features of the LN of KFD include irregular paracortical areas of coagulative necrosis with abundant karyorrhectic debris, with large numbers of different types of histiocytes at the margin of the necrotic areas [Fig.1]. Karryorhectic foci are formed by different cell types, predominantly histiocytes and plasmacytoid monocytes, but also immunoblasts and small and large lymphocytes. Neutrophils

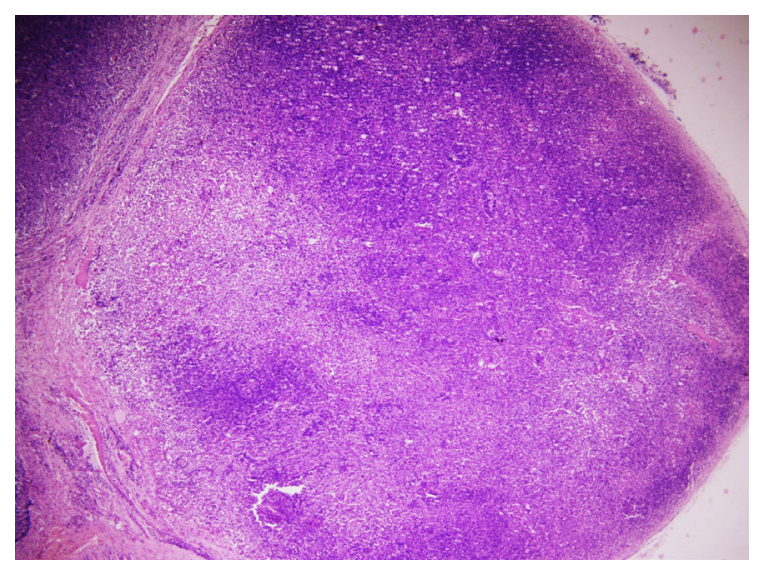

Fig.1: Large geographic areas of necrosis (Haematoxylin \& Eosin, 40x).

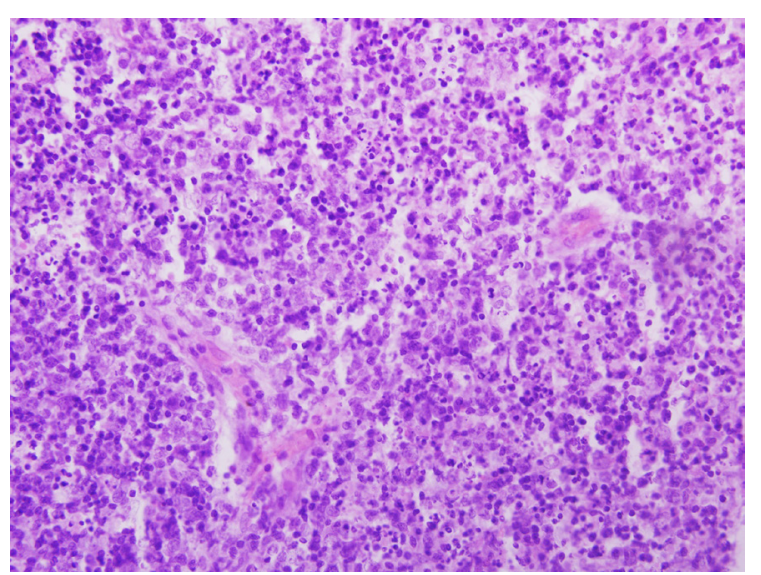

Fig.2: Histiocytes with admixed lymphocytes (Haematoxylin \& Eosin, 400x).

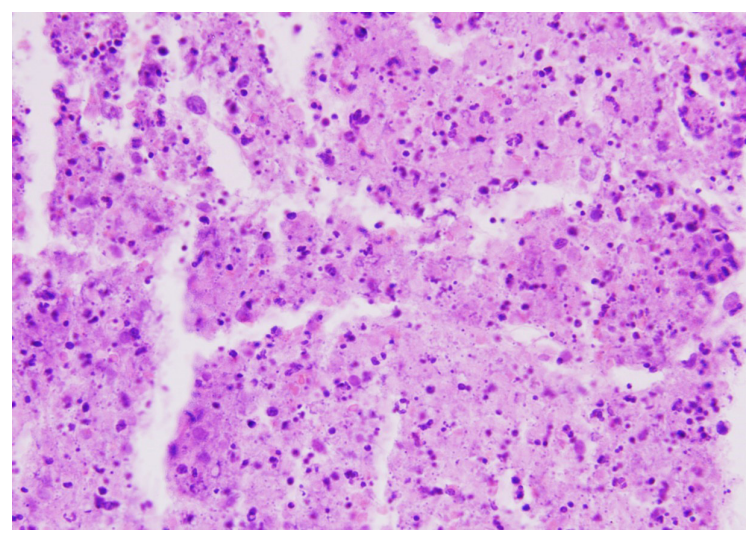

Fig.3: Foci of necrosis with interspersed fragmented nuclei and few intact lymphocytes. Neutrophils are absent (Haematoxylin \& Eosin, 400x). 
are characteristically absent and plasma cells are absent or scarce [Fig.3]. The minimum diagnostic criterion and the earliest features are the presence of paracortical clusters of plasmacytoid monocytes with interspersed karyorrhexis and crescentic histiocytes [8].

The classical presentation includes fever with lymphadenopathy. Sometimes fever can be accompanied by chills and rigors. The cervical group of LN predominantly involve especially the posterior triangle. An affected nodes may vary in size between 0.5 and $4 \mathrm{~cm}$ and may or may not be painful and tender [4,9]. Involvement of other LN sites is less common [10]. Painless cervical lymphadenopathy was the most common presenting features in an Indian case series reported by Supari $\mathrm{D}$ et al. Other constitutional features include fatigue, anorexia, sore throat, arthralgia, night sweats and skin rash [11]. There are certain cases of KFD that have presented as fever of unknown origin (FUO) [4].

The diagnosis is confirmed by histopathological examination of an affected LN. Biopsy is preferred to FNAC as FNAC doesn't show typical features like paracortical areas of necrosis, abundant karyorrhexis, with large numbers of different types of histiocytes at the margin of the necrotic areas. Clinically and histopathologically KFD resembles TB lymphadenitis, lymphoma and SLE. There are few reports where KFD had been wrongly diagnosed or treated as Hodgkin's lymphoma and TB [8]. Other histological differential diagnosis include plasmacytoid $\mathrm{T}$ cell leukemia, metastatic adenocarcinoma, infectious lymphadenitis and Kawasaki disease [4]. KFD should be considered as an important differential diagnosis in patients with lymphadenopathy.

It has been reported that among patients with proven KFD, weight loss, arthralgia, cutaneous manifestations, and ANA antibodies were associated with the development of SLE and a higher risk of recurrence [7]. Though our patient was positive for ANA, he remains asymptomatic without any features of SLE during 6 months of follow up.

Apart from symptomatic treatment, there is no specific treatment for KFD. Reassurance of the patient must not be forgotten. Immunosuppressant like corticosteroid, intravenous immunoglobulin might be helpful in life threatening conditions. Recurrence of the disease is possible but very rare [4].

\section{Conclusion}

KFD is a self-limiting cause of lymphadenopathy with fever which doesn't need any specific treatment except reassurance. In countries like India, where infectious diseases like TB are common causes of lymphadenopathy, the physician and pathologist must be aware of this disease entity and keep it among the differential diagnosis of peripheral lymphadenopathy, particularly in the young.

Contributors: SA: Concept, manuscript writing and supervison, proof reading, clinical care; VA: Literature search, manuscript writing; NK: Clinical care, pathology, and photographs.

Funding: None; Competing interests: None stated.

\section{References}

1. Kikuchi M. Lymphadenitis showing focal reticulum cell hyperplasia with nuclear debris and phagocytosis. Nippon Gakkai Zasshi. 1972;35:379-380.

2. Fujimoto Y, Kozima Y, Yamaguchi K. Cervical subacute necrotizing lymphadenitis: a new clinicopathological entity. Naika. 1972;20:920-927.

3. Kampitak T. Fatal Kikuchi-Fujimoto disease associated with SLE and hemophagocytic syndrome: a case report. Clin Rheumatol. 2008;27:1073-1075.

4. Bosch X, Guilabert A, Miquel R, Campo E. Enigmatic Kikuchi-Fujimoto disease: a comprehensive review. Am J Clin Pathol. 2004;122:141-152.

5. Lee S, Yoo JH, Lee SW. Kikuchi disease: differentiation from tuberculous lymphadenitis based on patterns of nodal necrosis on CT. AJNR Am J Neuroradiol. 2012;33:135-140.

6. Rosado FGN, Tang YW, Hasserjian RP, McClain CM, Wang B, Mosse CA. Kikuchi-Fujimoto lymphadenitis: role of parvovirus B-19, Epstein-Barr virus, human 
herpes virus 6 and human herpes virus 8. Hum Pathol. 2013;44:255-259.

7. Dumas G, Prendki V, Haroche J, Amoura Z, Cacoub P, Galicier L, et al. Kikuchi-Fujimoto disease: retrospective study of 91 cases and review of the literature. Medicine (Baltimore). 2014;93:372-382.

8. Tsang WYW, Chan JKC, Ng CS. Kikuchi's lymphadenitis: a morphologic analysis of 75 cases with special reference to unusual features. Am J Surg Pathol. 1994;18:219-231.

9. Dorfman RF, Berry GJ. Kikuchi'shistiocytic necrotizing lymphadenitis: an analysis of 108 cases with emphasis on differential diagnosis. Semin Diagnostic Pathol. 1998;5:329-345.

10. Vijayaraghavan R, Chandrashekar R, Saraswathi A, Belagavi CS. Kikuchi-Fujimoto's disease involving mesenteric nodes: a report and review of literature. BMJ Case Rep. 2011 Dec 1;2011.

11. Supari D, Ananthamurthy A. Kikuchi-fujimoto disease: a study of 24 cases. Indian J Otolaryngol Head Neck Surg. 2014;66:69-73. 